$\xi_{p}$

\title{
An M/G/1 Retrial Queue with Fluctuating Modes of Services
}

\author{
P. Rajadurai*, S. Venkatesh and K. Parameswari \\ Department of Mathematics, Srinivasa Ramanujan Centre, \\ Shanmugha Arts, Science, Technology \& Research Academy \\ (SASTRA Deemed to be University), \\ Kumbakonam, Tamilnadu, India. \\ *Corresponding author E-mail: psdurai17@gmail.com
}

\begin{abstract}
In this paper, we consider a single server retrial queueing system with working vacation and two classes of customers, which are priority customers and ordinary customers. The single server provides fluctuating modes (optional phases) of services. Using the method of Probability Generating Function (PGF) approach and supplementary variable technique, the steady state results are obtained.
\end{abstract}

Keywords: retrial queues, priority customers, working vacations.

\section{Introduction}

In queueing theory, vacation queues and retrial queues have been concentrated research topics in last many decades. For general models, we can find vacation models from Ke et al. [5] and retrial queues from Artalejo and Gomez-Corral [2]. More comprehensive review, readers may refer from [1], [3], [4-8]. In this paper, we have generalized the work of Gao [3] by including the concepts of fluctuating modes of services and re-service.

\section{Model Description}

"The detailed mathematical model description is given as follows: - The arrival Process: There are two types of customers arrive into the system: priority customers and ordinary customers. Priority customers have preemptive priorities over ordinary customers in service time of busy server. Assume that both types of customers arrive from outside the system according to three independent Poisson processes with rates $\lambda$ and $\delta$ respectively.

- The retrial process: We assume that there is no waiting space and therefore an arriving priority customer finds the server free; the customer begins its service immediately. At the arrival time of a priority customer, the server gives service for a priority customer or working vacations, the newly arriving priority customer will depart the system directly without service. While the regular busy server is working with an ordinary customer, the arriving priority customer will interrupt the service of the ordinary customer and the server begins its service immediately. We assume that when an ordinary customer is preempted by a priority customer, the ordinary customer who was just being served before starts the service of the priority customer and waits in the service area for the remaining service to complete.

- If an arriving ordinary customer finds the server is being busy or on working vacation, the arrivals join pool of blocked customers called an orbit in accordance with FCFS discipline. That is, only one customer at the head of the orbit queue is allowed access to the server. Then measured from the instant the server becomes free, an external potential priority customer or ordinary customer and a retrial ordinary customer compete to entire the server. Inter-retrial times have an arbitrary distribution $R(t)$ with corresponding Laplace Stieltijes Transform

(LST) $R^{*}(\vartheta)$. The retrial ordinary customer is required to give up the attempt for service if an external priority customer or ordinary customer arrives first. In that case, the retrial ordinary customer goes back to its position in the retrial queue.

- The regular and fluctuating modes of service process: Whenever a new primary (priority) customer or retry customer arrives at the server idle state then the server immediately starts normal service for the arrivals. In this model, we assume that a single server provides $k$ - optional phases of service in succession. The First Phase Service (FPS) is followed by $i(i=1,2, \ldots, k)$ phases of service. After completion of $i^{\text {th }}$ phase of service the customer may go to $(i+1)^{\text {th }}$ phase with probability $p_{i}$ or with probability $r_{i}$ may re-join into $i^{\text {th }}$ phase immediately to get a same service again (immediate feedback) or may leave the system with probability 1 $p_{i}-r_{i}=q_{i}$ for $(i=1,2, \ldots, k)$, if the service is successful. After completion $k^{\text {th }}$ phase service the customers may re-join to $k^{\text {th }}$ phase with probability $r_{k}$ or leaves the system with probability $q_{k}=1-r_{k}$. The service time of priority customers follows a general distribution and denoted by the random variable $S_{p}$ with distribution function $S_{p}(t)$ having $\operatorname{LST} S_{p}^{*}(\vartheta)$ and the first and second moments are $\beta_{p}^{(1)}$ and $\beta_{p}^{(2)}$. The service time of ordinary customers follows a general distribution for all the phases. It is denoted by the random variable $S_{i}$ with distribution function $S_{i}(t)$

having $\operatorname{LST} S_{i}^{*}(\vartheta)$, first and second moments are $\beta_{i}^{(1)}$ and $\beta_{i}^{(2)}$ for $(i=1,2, \ldots, k)$.

- The working vacation process: The server begins a working vacation each time when the orbit becomes empty and the vacation time follows an exponential distribution with parameter $\theta$ During a vacation period if any customer arrives, the server gives service at a lower speed service rate. If any customers are in the orbit at a lower speed service completion instant in the vacation period then the server will stop the vacation and come back to the 
normal busy period which means that vacation interruption happens. Otherwise, it continuous the vacation. When a vacation ends and if there are customers in the orbit then the server switches to the normal working level. During the working vacation period, the service time follows a general random variable $S_{v}$ with distribution function $S_{v}(t)$ having $\operatorname{LST} S_{v}^{*}(\vartheta)$ and the first moment is given by $S_{v}^{*^{\prime}}(\theta)=\int_{0}^{\infty} x e^{-\theta x} d S_{v}(x)$.

- Various stochastic processes involved in the system are assumed to be independent of each other.

- Throughout the rest of the paper, we denote by $\bar{F}(x)=1-F(x)$ the tail of distribution function $F(x)$. We also denote $F^{*}(x)=\int_{0}^{\infty} e^{-s x} d F(x)$, the LST of $F(x) \quad$ and $\tilde{F}(s)=\int_{0}^{\infty} e^{-s x} F(x) d x$ to be the Laplace transform of $F(x)$ and we assume the notation $\bar{F}^{*}(s)=\frac{1-F^{*}(s)}{s}$ and the expected value is $\beta^{(1)}=\sum_{i=1}^{k} \Theta_{i-1} \beta_{i}^{(1)}$. where $\Theta_{i}=p_{1} p_{2} \ldots p_{i}$ and $\Theta_{0}=1$.”

\section{Steady state analysis}

In this section, we derived the steady state difference-differential equations and the PGF for the server states.

"In steady state, we consider that $R(0)=0, R(\infty)=1, S_{p}(0)=0$, $S_{p}(\infty)=1, S_{v}(0)=0, S_{v}(\infty)=1$ are continuous at $x=0$ and $S_{i}(0)=0$, $S_{i}(\infty)=1$ (for $\left.i=1,2, \ldots, k\right)$ are continuous at $y=0$. The conditional completion rates for retrial, regular service for priority customers, ordinary customers and lower rates service are as follows $a(x), \mu_{p}(x), \mu_{i}(y)$ and $\mu_{v}(x)$ respectively.

$(\lambda+\delta+\theta) P_{0}=\theta P_{0}+\int_{0}^{\infty} \Pi_{1,0}(x) \mu_{p}(x) d x+\sum_{i=1}^{k-1} q_{i} \int_{0}^{\infty} P_{i, 0}(y) \mu_{i}(y) d y+\left(1-r_{k}\right) \int_{0}^{\infty} P_{k, 0}(y) \mu_{k}(y) d y+\sum_{i=1}^{k} \int_{0}^{\infty} \Omega_{i, 0}(y) \mu_{i}(y) d y+\int_{0}^{\infty} Q_{v, 0}(x) \mu_{v}(x) d x$

$\frac{d R_{n}(x)}{d x}+(\lambda+\delta+a(x)) R_{n}(x)=0, n \geq 1$.

$\frac{d \Pi_{1, n}(x)}{d x}+\left(\lambda+\mu_{p}(x)\right) \Pi_{1, n}(x)=\lambda \Pi_{1, n-1}(x), n \geq 1$.

$\frac{\partial \Pi_{2, n}(x, y)}{\partial x}+\left(\lambda+\mu_{p}(x)\right) \Pi_{2, n}(x, y)=\lambda \Pi_{2, n-1}(x, y), n \geq 1$.

$\frac{d P_{i, n}(y)}{d y}+\left(\lambda+\delta+\mu_{i}(y)\right) P_{i, n}(y)=\lambda P_{i, n-1}(y)+\int_{0}^{\infty} \Pi_{2, n}(x, y) \mu_{p}(x) d x, n \geq 1$.

$\frac{d \Omega_{i, n}(y)}{d y}+\left(\lambda+\delta+\mu_{i}(y)\right) \Omega_{i, n}(y)=\lambda \Omega_{i, n-1}(y)+\int_{0}^{\infty} \Pi_{2, n}(x, y) \mu_{p}(x) d x, n \geq 1$.

$\frac{d Q_{v, n}(x)}{d x}+\left(\lambda+\theta+\mu_{v}(x)\right) Q_{v, n}(x)=\lambda Q_{v, n-1}(x), n \geq 1$. 
The steady state boundary conditions at $x=0$ and $y=0$ are

$R_{n}(0)=\sum_{i=1}^{k-1} q_{i} \int_{0}^{\infty} P_{i, n}(y) \mu_{i}(y) d y+\left(1-r_{k}\right) \int_{0}^{\infty} P_{k, n}(y) \mu_{k}(y) d y+\sum_{i=1}^{k} \int_{0}^{\infty} \Omega_{i, n}(y) \mu_{i}(y) d x+\int_{0}^{\infty} Q_{v, n}(x) \mu_{v}(x) d x+\int_{0}^{\infty} \Pi_{1, n}(x) \mu_{p}(x) d x, n \geq 1$.

$\Pi_{1, n}(0)=\delta \int_{0}^{\infty} R_{n}(x) d x, n \geq 1$

$\Pi_{2, n}(0)=\delta\left(P_{i, n}(y)+\Omega_{i, n}(y)\right), n \geq 0,(1=1,2, \ldots, k)$.

$P_{1, n}(0)=\int_{0}^{\infty} R_{n+1}(x) a(x) d x+\lambda \int_{0}^{\infty} R_{n}(x) d x+\theta \int_{0}^{\infty} Q_{v, n}(x) d x, n=0$.

$P_{i, n}(0)=P_{i-1} \int_{0}^{\infty} P_{i-1, n}(y) \mu_{i-1}(y) d y, n \geq 0,(i=1,2, \ldots, k)$.

$\Omega_{i, n}(0)=r_{i} \int_{0}^{\infty} P_{i, n}(y) \mu_{i}(y) d y, n \geq 0,(i=1,2, \ldots, k)$.

$Q_{v, n}(0)=(\lambda+\delta) P_{0}, n=0$.

The normalizing condition is

$P_{0}+\sum_{n=1}^{\infty} \int_{0}^{\infty} R_{n}(x) d x+\sum_{n=0}^{\infty}\left(\int_{0}^{\infty} \Pi_{1, n}(x) d x+\int_{0}^{\infty} Q_{v, n}(x) d x+\int_{0}^{\infty} \int_{0}^{\infty} \Pi_{2, n}(x, y) d x d y+\sum_{i=1}^{k}\left(\int_{0}^{\infty} \Omega_{i, n}(y) d y+\int_{0}^{\infty} P_{i, n}(y) d y\right)\right)=1$

\subsection{The steady state solution}

The PGF technique is used here to obtain the steady state solution and to solve the above equations, the GF for $|z| \leq 1$, for $(i=$ $1,2, \ldots, k)$ as follows:

$$
\begin{aligned}
& R(x, z)=\sum_{n=1}^{\infty} R_{n}(x) z^{n} ; \Pi_{1}(x, z)=\sum_{n=0}^{\infty} \Pi_{1, n}(x) z^{n} ; \Pi_{2}(x, y, z)=\sum_{n=0}^{\infty} \Pi_{2, n}(x, y) z^{n} ; \\
& P_{i}(y, z)=\sum_{n=0}^{\infty} P_{i, n}(y) z^{n} ; \Omega_{i}(y, z)=\sum_{n=0}^{\infty} \Omega_{i, n}(y) z^{n} ; Q_{v}(x, z)=\sum_{n=0}^{\infty} Q_{v, n}(x) z^{n} ; \\
& R(z)=\frac{z(\lambda+\delta) Q_{0}\left(\overline{R^{*}}(\lambda+\delta)\left[\left(S_{v}^{*}\left(A_{v}(z)\right)-1\right)+V(z)\left(\sum_{i=1}^{k}\left(q_{i}+r_{i} S_{i}^{*}\left(A_{i}(z)\right)\right)\right) \Theta_{i-1} B_{i}^{*}\left(A_{i}(z)\right)\right]\right)}{z-\left(R^{*}(\lambda+\delta)+\lambda z \overline{R^{*}}(\lambda+\delta)\right) \sum_{i=1}^{k}\left(q_{i}+r_{i} S_{i}^{*}\left(A_{i}(z)\right)\right) \Theta_{i-1} S_{i}^{*}\left(A_{i}(z)\right)-z \overline{R^{*}}(\lambda+\delta) S_{p}^{*}\left(A_{p}(z)\right)} \\
& \Pi_{1}(z)=\frac{z(\lambda+\delta) Q_{0} \delta \overline{R^{*}}(\lambda+\delta) \overline{S_{p}^{*}}\left(A_{p}(z)\right)\left[\left(S_{v}^{*}\left(A_{v}(z)\right)-1\right)+V(z)\left(\sum_{i=1}^{k}\left(q_{i}+r_{i} S_{i}^{*}\left(A_{i}(z)\right)\right)\right) \Theta_{i-1} B_{i}^{*}\left(A_{i}(z)\right)\right]}{z-\left(R^{*}(\lambda+\delta)+\lambda z \overline{R^{*}}(\lambda+\delta)\right) \sum_{i=1}^{k}\left(q_{i}+r_{i} S_{i}^{*}\left(A_{i}(z)\right)\right) \Theta_{i-1} S_{i}^{*}\left(A_{i}(z)\right)-z \delta \overline{R^{*}}(\lambda+\delta) S_{p}^{*}\left(A_{p}(z)\right)} \\
& \Pi_{2}(z)=(\lambda+\delta) Q_{0} \delta\left(1+r_{i} S_{i}^{*}\left(A_{i}(z)\right)\right) \overline{S_{p}^{*}}\left(A_{p}(z)\right) \overline{S_{i}^{*}}\left(A_{i}(z)\right) \Theta_{i-1} \\
& \times \frac{B_{i}^{*}\left(A_{i}(z)\right)\left[\left(S_{v}^{*}\left(A_{v}(z)\right)-1\right) R(z)+z V(z)\left(1-\delta \overline{R^{*}}(\lambda+\delta) S_{p}^{*}\left(A_{p}(z)\right)\right)\right]}{z-\left(R^{*}(\lambda+\delta)+\lambda z \overline{R^{*}}(\lambda+\delta)\right) \sum_{i=1}^{k}\left(q_{i}+r_{i} S_{i}^{*}\left(A_{i}(z)\right)\right) \Theta_{i-1} S_{i}^{*}\left(A_{i}(z)\right)-z \overline{R^{*}}(\lambda+\delta) S_{p}^{*}\left(A_{p}(z)\right)}
\end{aligned}
$$

Multiplying the equation (2) - (14) by $z^{n}$ and summing over $n,(n=$ $0,1,2, \ldots)$. Solving the partial differential equations then the limiting PGFs $R(x, z), \Pi_{1}(x, z), P_{i}(y, z), \Pi_{2}(x, y, z), \Omega_{i}(y, z)$ and $Q_{v}(x, z) "$.

"Theorem 3.1 Under the stability condition $\rho<R^{*}(\lambda+\delta)$, the stationary joint distributions of the number of customers in the orbit when server being idle, busy on priority customer, ordinary customer, re-service in ordinary customer and on working vaca- 


$$
\begin{aligned}
& P_{i}(z)=(\lambda+\delta) Q_{0} \Theta_{i-1} B_{i}^{*}\left(A_{i}(z)\right) \overline{S_{i}^{*}}\left(A_{i}(z)\right) \\
& \times \frac{\left[\left(S_{v}^{*}\left(A_{v}(z)\right)-1\right) R(z)+z V(z)\left(1-\delta \overline{R^{*}}(\lambda+\delta)\left(S_{p}^{*}\left(A_{p}(z)\right)\right)\right]\right.}{z-\left(R^{*}(\lambda+\delta)+\lambda z \overline{R^{*}}(\lambda+\delta)\right) \sum_{i=1}^{k}\left(q_{i}+r_{i} S_{i}^{*}\left(A_{i}(z)\right)\right) \Theta_{i-1} S_{i}^{*}\left(A_{i}(z)\right)-z \delta \overline{R^{*}}(\lambda+\delta) S_{p}^{*}\left(A_{p}(z)\right)} \\
& \Omega_{i}(z)=(\lambda+\delta) Q_{0} r_{i} S_{i}^{*}\left(A_{i}(z)\right) \Theta_{i-1} B_{i}^{*}\left(A_{i}(z)\right) \overline{S_{i}^{*}}\left(A_{i}(z)\right) \\
& \times \frac{\left[\left(S_{v}^{*}\left(A_{v}(z)\right)-1\right) R(z)+z V(z)\left(1-\delta \overline{R^{*}}(\lambda+\delta)\left(S_{p}^{*}\left(A_{p}(z)\right)\right)\right]\right.}{z-\left(R^{*}(\lambda+\delta)+\lambda z \overline{R^{*}}(\lambda+\delta)\right) \sum_{i=1}^{k}\left(q_{i}+r_{i} S_{i}^{*}\left(A_{i}(z)\right)\right) \Theta_{i-1} S_{i}^{*}\left(A_{i}(z)\right)-z \delta \overline{R^{*}}(\lambda+\delta) S_{p}^{*}\left(A_{p}(z)\right)} \\
& Q_{v}(z)=\left\{\frac{(\lambda+\delta) Q_{0} V(z)}{\theta}\right\} \\
& P_{0}=\frac{R^{*}(\lambda+\delta)-\rho}{\eta}
\end{aligned}
$$

Where

$$
\begin{aligned}
& \eta=R^{*}(\lambda+\delta)-\rho+((\lambda+\delta) / \theta)\left(1-S_{v}^{*}(\theta)\right) \\
& \quad \times\left\{R^{*}(\lambda+\delta)(1+(\lambda+\delta) / \theta) \sum_{i=1}^{k} \Theta_{i-1} \lambda\left(1+r_{i}\right) \beta_{i}^{(1)}\left(1+\delta \beta_{p}^{(1)}\right)\right\} \\
& \rho=\sum_{i=1}^{k} \Theta_{i-1} M_{1 i}+\lambda \sum_{i=1}^{k} r_{i} \Theta_{i-1} \beta_{i}^{(1)}-\sum_{i=1}^{k-1} \Theta_{i} M_{1 i}+\delta \overline{R^{*}}(\lambda+\delta) \lambda \beta_{p}^{(1)} \\
& V(z)=\frac{\theta\left(1-S_{v}^{*}\left(A_{v}(z)\right)\right)}{\theta+\lambda(1-z)} ; \overline{S_{p}^{*}}\left(A_{p}(z)\right)=\frac{\left(1-\overline{S_{p}^{*}}\left(A_{p}(z)\right)\right)}{A_{p}(z)} \\
& A_{v}(z)=(\theta+\lambda(1-z)) ; \\
& \overline{S_{i}^{*}}\left(A_{i}(z)\right)=\frac{\left(1-\overline{S_{i}^{*}}\left(A_{i}(z)\right)\right)}{A_{i}(z)} ; A_{p}(z)=\lambda(1-z) ; \\
& A_{b}(z)=\left(A_{p}(z)+\delta\left(1-S_{p}^{*}\left(A_{p}(z)\right)\right)\right) .
\end{aligned}
$$

Proof Integrating the limiting PGF's with respect to $x$ and $y$, then we get the equations (16-(21). The value of $P_{0}$ can be determined using the normalizing condition. By setting $z=1$ in (16)-(21) and applying L-Hospitals rule whenever necessary and we get

$P_{0}+R(1)+Q_{v}(1)+\Pi_{1}(1)+\Pi_{2}(1)+\sum_{i=1}^{k}\left(P_{i}(1)+\Omega_{i}(1)\right)=1$.

Corollary 3.1: If the system satisfies the stability condition,

(i) The PGF of number of customers in the system $\left(K_{s}(z)\right)$

$$
K_{s}(z)=P_{0}+R(z)+z\left(Q_{v}(z)+\Pi_{1}(z)+\Pi_{2}(z)+\sum_{i=1}^{k}\left(P_{i}(z)+\Omega_{i}(z)\right)\right) \text {. }
$$

(ii) The PGF of the number of customer in the orbit $\left(K_{o}(z)\right)$

$$
K_{o}(z)=P_{0}+R(z)+Q_{v}(z)+\Pi_{1}(z)+\Pi_{2}(z)+\sum_{i=1}^{k}\left(P_{i}(z)+\Omega_{i}(z)\right) .
$$

\section{Performance measures}

The excepted number of customers in the orbit $\left(L_{q}\right)$

$$
L_{q}=K_{o}^{\prime}(1)=\lim _{z \rightarrow 1} \frac{d}{d z} K_{o}(z)
$$

The excepted number of customers in the system $\left(L_{s}\right)$

$$
L_{s}=K_{s}^{\prime}(1)=\lim _{z \rightarrow 1} \frac{d}{d z} K_{s}(z)
$$

The average time a customer spends in the system $\left(W_{s}\right)$ $L_{s}=\lambda W_{s}$

The average time a customer spends in the queue $\left(W_{q}\right)$ $L_{q}=\lambda W_{q}$."

\section{Concluding remarks}

In this paper, we analysed a single server retrial queueing system with two classes of customers and multiple working vacations. The steady state results and system performance measures are found. This model identifies application in telecommunication system and telephone consultation of medical service systems.

\section{References}

[1] Arivudainambi D, Godhandaraman P \& Rajadurai P (2014) Performance analysis of a single server retrial queue with working vacation, OPSEARCH 51, 434-462

[2] Artalejo J \& Gomez-Corral A (2008), Retrial Queueing Systems, Springer, Berlin, Germany.

[3] Gao S (2015), A preemptive priority retrial queue with two classes of customers and general retrial times. Operational Research 15 , 233-251.

[4] Gao S, Wang J \& Li W (2014), An M/G/1 retrial queue with general retrial times, working vacations and vacation interruption. Asia-Pacific Journal of Operational Research 31, 6-31.

[5] Ke JC, Wu CU \& Zhang ZG (2010), Recent developments in vacation models: a short survey. International Journal of Operations Research 7, 3-8.

[6] Rajadurai P (2018), Sensitivity analysis of an M/G/1 retrial queueing system with disaster under working vacations and working breakdowns. RAIRO operations research 52, 35-54.

[7] Rajadurai P, Saravanarajan MC \& Chandrasekaran VM (2014), Analysis of an $\mathrm{M}^{[\mathrm{X}]} /\left(\mathrm{G}_{1}, \mathrm{G}_{2}\right) / 1$ retrial queueing system with balking, optional re-service under modified vacation policy and service interruption, Ain Shams Engineering Journal 5, 935-950.

[8] Servi LD \& Finn SG (2002), M/M/1 queues with working vacations Performance Evaluation 50, 41-52. 